\title{
AVALIAÇÃO DA QUALIDADE DE SEDIMENTOS - ESTUDO DE CASO: SUB-BACIA DO RIBEIRÃO ESPÍRITO SANTO, AFLUENTE DO RIO SÃO FRANCISCO
}

\author{
Vanessa Kelly Saraiva \\ Instituto Mineiro de Gestão das Águas, Rua Espírito Santo, 495, 30160-030 Belo Horizonte - MG, Brasil \\ Marcos Roberto Lopes do Nascimento \\ Comissão Nacional de Energia Nuclear, Rodovia Poços de Caldas, km 13, 37701-970 Poços de Caldas - MG, Brasil \\ Helena Eugênia Leonhardt Palmieri e Vanusa Maria Feliciano Jacomino* \\ Comissão Nacional de Energia Nuclear, Centro de Desenvolvimento da Tecnologia Nuclear, Av. Pres. Antônio Carlos, 6.627, \\ Campus da UFMG, 31270-901 Belo Horizonte - MG, Brasil
}

Recebido em 14/5/08; aceito em 13/4/09; publicado na web em 30/9/09

\begin{abstract}
EVALUATION OF SEDIMENT QUALITY - CASE STUDY: SUB-WATERSHED OF ESPÍRITO SANTO STREAM, AFFLUENT OF THE SÃO FRANCISCO RIVER. This study evaluated the environmental impact resulting from surface water and sediment contamination by metals in a watershed affected by a tailing basin that controls effluents coming from a zinc-ore beneficiation plant. The studies combined assessments of sediment chemistry (exceedances of sediment quality guidelines), benthic assemblage structure and acute and chronic ecotoxicity. The results showed that the levels of metal contamination in sediments are not yet enough to cause deleterious effects to the biota. However, the ecotoxicity tests indicated the occurrence of chronic effects, demonstrating that other factors, as the use of fertilizers, could also be a source of contamination.
\end{abstract}

Keywords: sediments; acid volatile sulfite; benthos.

\section{INTRODUÇÃO}

O crescimento da população mundial estimula o aumento das atividades industriais, comerciais e agropecuárias. O desenvolvimento dessas atividades, de forma não sustentável, contribui para a contaminação dos recursos hídricos superficiais e subterrâneos em decorrência do lançamento de rejeitos contendo metais, matérias orgânicas, fertilizantes, pesticidas, óleos, percolados tóxicos de lixões, entre outros. Consequentemente, além da escassez, a sociedade mundial está se deparando com a queda da qualidade das águas em taxas muito acentuadas. Para combater esta ameaça é necessário avaliar a magnitude e a natureza dos problemas envolvidos e, portanto, estudos que contribuam para um melhor entendimento da relação causa-efeito, entre os processos produtivos e seu impacto ambiental em ecossistemas aquáticos são cada vez mais importantes e indispensáveis. ${ }^{1}$

Como resultados destes processos, os estudos de avaliação da qualidade da água têm passado por mudanças substanciais que incluem a avaliação da qualidade dos sedimentos, um compartimento que era, até bem pouco tempo, apenas considerado como um acumulador de nutrientes e de uma variedade de contaminantes. ${ }^{2}$ Pesquisas relacionadas à troca de espécies químicas dissolvidas entre o sedimento e a coluna d'água têm demonstrado que esse compartimento deve ser considerado como um ambiente de trocas e/ou interações dessas espécies com a coluna d'água e a biota residente. ${ }^{3}$

Uma variedade de fatores físicos, químicos e biológicos controla a mobilidade dos contaminantes presentes nos sedimentos, tais como ressuspensão pelo revolvimento, sorção, alterações de $\mathrm{pH}$ e potencial redox, entre outros. ${ }^{4}$ Dependendo da estabilidade físico-química do ambiente esses contaminantes podem permanecer ligados por longo tempo aos suportes sedimentares, como os metais em ambientes anóxicos ou, então, sofrer degradação como a maior parte dos compostos orgânicos (hidrocarbonetos e organoclorados). Uma vez depositados nos sedimentos, os contaminantes podem sofrer modificações quí-

*e-mail: vmfj@cdtn.br micas e biológicas que os disponibilizam à biota (micro-organismos, flora e fauna de um ecossistema).

Nos últimos anos têm sido desenvolvidas metodologias para uma avaliação mais abrangente da qualidade de sedimentos que adotam abordagens integradas combinando evidências físicas, químicas, biológicas e toxicológicas. A abordagem que integra dados químicos, biológicos e ecotoxicológicos, denominada tríade da qualidade de sedimentos, é considerada uma das melhores para o estudo de sistemas aquáticos impactados por efluentes antropogênicos. ${ }^{5}$ Nesse caso, dados físicos e químicos (conteúdo de matéria orgânica, concentração de metais, entre outros) e biológicos (ecotoxicidade e biodiversidade) são combinados para gerar um perfil de degradação de cada unidade amostral.

De forma a avaliar o impacto ambiental decorrente da contaminação de sedimentos e águas superficiais por metais nas sub-bacias do ribeirão Espírito Santo e córrego Lavagem, afluentes direto e indireto do rio São Francisco na região de Três Marias - MG, foi instalada uma rede dirigida para o levantamento de dados referentes à qualidade das águas e dos sedimentos na região. Vale ressaltar que essas sub-bacias são afetadas em decorrência da implantação de uma bacia de rejeitos oriundos da produção de zinco e os principais impactos presentes na região incluem, além da barragem de rejeitos, atividades de pasto e plantio em pequenas propriedades.

As pesquisas foram direcionadas à caracterização biogeoquímica dos sedimentos por meio da determinação das concentrações de sulfetos volatilizáveis por acidificação (SVA) e da determinação da fração de metais associada aos SVA, aos carbonatos, aos óxidos amorfos, adsorvidos às superfícies das partículas sedimentares e frações mais lábeis da matéria orgânica nos sedimentos totais, denominada metais extraídos simultaneamente (MES). Os dados levantados foram interpretados em associação aos resultados da estrutura da comunidade bentônica e testes de ecotoxicidade. Os estudos incluíram, ainda, a realização de análises das variáveis físico-químicas e da concentração de metais nas amostras de águas superficiais; análises granulométricas e determinação do teor de matéria orgânica em amostras de sedimentos. 
O presente trabalho tem por objetivo principal avaliar a qualidade ambiental da área estudada, com base em critérios geoquímicos, biológicos e ecotoxicológicos, bem como discutir os resultados obtidos em cada uma das campanhas de amostragem.

\section{PARTE EXPERIMENTAL}

\section{Seleção dos pontos de amostragem}

A sub-bacia selecionada para este estudo sofre com a influência de uma bacia de rejeitos oriundos da produção de zinco, a qual foi implantada em 2002 nas cabeceiras do córrego Lavagem. Esse curso de água deságua no ribeirão Espírito Santo que, por sua vez, é afluente direto do rio São Francisco, na região de Três Marias, MG.

A fim de averiguar a possível contaminação das águas e sedimentos do ribeirão Espírito Santo e do seu afluente, o córrego Lavagem, foram definidos quatro pontos de amostragem, os quais estão relacionados na Tabela 1 .

\section{Amostragem das águas superficiais e sedimentos}

No total foram realizadas quatro campanhas de amostragem, uma em novembro de 2005 (período chuvoso) e as outras três em 2006, nos meses de fevereiro (período chuvoso), agosto (período de seca) e novembro (período chuvoso). Para evitar a contaminação das amostras de água e sedimentos foram utilizados frascos de polietileno e sacos plásticos descartáveis.

As campanhas de nov/05 e fev/06 foram consideradas incompletas, uma vez que a primeira foi feita para uma avaliação preliminar da qualidade da água e sedimentos, para determinação de dados sobre a concentração de metais na água e sedimento e determinação da granulometria, e a segunda foi feita para a avaliação da estrutura da comunidade bentônica. Ressalta-se que para análise da estrutura da comunidade bentônica foi feita apenas uma coleta, pois as análises em laboratório são extremamente morosas. Além disso, os organismos bentônicos possuem um ciclo de vida longo e, portanto, num intervalo de tempo relativamente curto, não são observadas variações significativas na sua população. Os resultados dessa campanha indicaram a existência de uma grande variedade de organismos bentônicos na região em estudo.

As campanhas realizadas nos meses de agosto e novembro de 2006 foram consideradas completas, uma vez que foram coletadas amostras de sedimentos para levantamento de dados sobre a concentração de metais totais, determinação do conteúdo de matéria orgânica, das concentrações de sulfetos volatilizáveis por acidificação (SVA) e dos metais extraídos simultaneamente (MES), avaliação das toxicidades aguda e crônica e determinação da granulometria. Foram também coletadas amostras de água superficial para determinação dos parâmetros físico-químicos, análise dos metais $(\mathrm{Cd}, \mathrm{Cu}, \mathrm{Pb}, \mathrm{Ni}$ e Zn) e para ensaios de ecotoxicidade.

Imediatamente após a coleta, as amostras de sedimentos e água superficial destinadas às análises químicas e de ecotoxicidade foram acondicionadas em sacos plásticos e mantidas em caixas de isopor com gelo. As amostras de sedimento superficial (0-15 cm de profundidade) foram coletadas por meio de uma concha de plástico. Para as análises das águas foi coletado um volume de $1 \mathrm{~L}$ em cada ponto com a ajuda de uma jarra graduada em polietileno.

Para avaliação da estrutura da comunidade bentônica, em cada ponto amostral, foram coletadas três alíquotas de sedimento superficial (0-15 $\mathrm{cm}$ de profundidade) com a ajuda de um amostrador Surber. Ainda no campo, logo após a coleta, as amostras foram fixadas com formol $10 \%$ e mantidas em sacos plásticos até seu processamento em laboratório.

\section{Preparação das amostras}

\section{Água superficial}

Os parâmetros analisados nas águas superficiais in loco foram temperatura, condutividade, sólidos totais dissolvidos e potencial hidrogeniônico - pH. No laboratório foram avaliados os parâmetros oxigênio dissolvido - OD e os metais cádmio total, cobre dissolvido, chumbo total, níquel total e zinco total. As amostras de água de superfície destinadas à quantificação dos metais citados anteriormente foram preservadas com ácido nítrico concentrado (65\%). No caso específico das análises de cobre dissolvido, devido a uma exigência da Resolução CONAMA 357, ${ }^{6}$ as amostras foram filtradas com membranas de éster de celulose de $0,45 \mu \mathrm{m}$ de porosidade, acidificadas $\left(\mathrm{HNO}_{3}, 65 \%\right)$ e, a seguir, enviadas para análise.

\section{Sedimentos}

Para as análises de SVA e MES, logo após a coleta, os sedimentos foram refrigerados em caixas de isopor com gelo e processados ainda úmidas em laboratório. Para determinação da concentração de metais totais nas amostras de sedimentos as amostras foram inicialmente secas à temperatura ambiente, passadas por uma peneira de 6 mesh para retirada de detritos, tais como folhas e galhos, homogeneizadas e, posteriormente, pulverizadas.

O método utilizado para a extração dos metais das amostras de sedimento foi o 3050B da Agência Ambiental dos Estados Unidos. ${ }^{7}$ Esse método não é uma técnica para digestão total dos elementos, mas uma digestão ácida capaz de solubilizar elementos que poderão ser ambientalmente disponíveis. Em resumo, consiste na pesagem de aproximadamente $1 \mathrm{~g}$ de amostra seca com acidificação a quente com ácido nítrico e peróxido de hidrogênio, por repetidas vezes. Ao final adiciona-se ácido clorídrico, sempre sob aquecimento. Após a filtragem das amostras com membranas de éster de celulose de $0,45 \mu \mathrm{m}$ de porosidade, as mesmas foram enviadas ao Laboratório do Setor Analítico da Comissão Nacional de Energia Nuclear em Poços de Caldas para determinação das concentrações de $\mathrm{Cd}, \mathrm{Cu}$, $\mathrm{Pb}$, Ni e $\mathrm{Zn}$. Ressalta-se que foram realizadas duas replicatas da extração dos sedimentos.

\section{Métodos analíticos}

\section{Determinação dos parâmetros físico-químicos}

A determinação do oxigênio dissolvido (OD) nas águas superficiais foi realizada pelo Método de Winkler. ${ }^{8}$ Para avaliar os parâmetros condutividade elétrica e sólidos totais dissolvidos foi utilizado um condutivímetro e para análise de $\mathrm{pH}$, um pHmêtro, ambos da marca Digimed.

Tabela 1. Descrição das estações de amostragem na sub-bacia do ribeirão Espírito Santo

\begin{tabular}{llll}
\hline Estação & Descrição & UTM (X) & UTM (Y) \\
\hline CL002 & Córrego Lavagem a montante de sua foz no ribeirão Espírito Santo & 0479433 & 7991966 \\
ES001 & Ribeirão Espírito Santo a montante da confluência com o córrego Lavagem & 0480172 & 7991352 \\
ES002 & Ribeirão Espírito Santo a jusante da confluência com o córrego Lavagem & 0479870 & 7992800 \\
ES003 & Ribeirão Espírito Santo a montante de sua foz no rio São Francisco & 0479995 & 7994036 \\
\hline
\end{tabular}




\section{Análise granulométrica}

A determinação da granulometria das amostras de sedimento foi feita pela técnica do peneiramento, conforme metodologia proposta pela Associação Brasileira de Normas Técnicas (ABNT). ${ }^{9}$

\section{Análise do conteúdo de matéria orgânica presente nos sedimentos}

Para determinação do conteúdo de matéria orgânica nos sedimentos as amostras foram inicialmente secas em estufa a aproximadamente $60^{\circ} \mathrm{C}$ e maceradas. A seguir, os cadinhos utilizados na pesagem dos sedimentos foram colocados em mufla a aproximadamente $300{ }^{\circ} \mathrm{C}$ para retirar a umidade, pesados e suas respectivas massas anotadas.

Aproximadamente $0,3 \mathrm{~g}$ de sedimento foram levadas à mufla para queima a uma temperatura de aproximadamente $550{ }^{\circ} \mathrm{C}$ por $3 \mathrm{~h}$. Posteriormente, as amostras foram novamente pesadas para determinação da quantidade de massa queimada, correspondente à matéria orgânica presente nas amostras.

\section{Determinação de metais}

No presente estudo a determinação dos metais $\mathrm{Cd}, \mathrm{Cu}, \mathrm{Pb}, \mathrm{Ni}$ e Zn foi feita pelas técnicas de espectrometria de emissão atômica com plasma indutivamente acoplado (ICP-AES) e/ou espectrometria de absorção atômica com forno de grafite (ET-AAS). A escolha da técnica foi em função de seus limites de detecção, de acordo com os padrões de qualidade da água recomendados pela legislação ambiental. ${ }^{6}$

\section{Análise de sulfetos volatilizáveis por acidificação (SVA) e metais extraídos simultaneamente (MES)}

A extração dos sulfetos volatilizáveis por acidificação (SVA) e da fração de metais associada aos SVA, conhecida como metais extraídos simultaneamente (MES), nos sedimentos foi realizada pelo método desenvolvido por Allen et al. ${ }^{10}$

O método consiste em um sistema de reação onde aproximadamente 4 a $5 \mathrm{~g}$ de sedimento úmido são misturados a $100 \mathrm{~mL}$ de água deionizada (purificador de água MilliPore, Milli-Q ${ }^{\circledR}$ ) em um balão de reação. A seguir, o balão é fechado e a solução é constantemente agitada e submetida a um fluxo de nitrogênio, borbulhado lentamente por aproximadamente 15 min. Em seguida, são introduzidos $20 \mathrm{~mL}$ de ácido clorídrico $(\mathrm{HCl})$ $6 \mathrm{~mol} \mathrm{~L}^{-1}$, previamente desaerado. Os sulfetos, liberados na forma de ácido sulfídrico $\left(\mathrm{H}_{2} \mathrm{~S}\right)$ são arrastados por meio do fluxo de nitrogênio até os frascos receptores que contêm $80 \mathrm{~mL}$ de solução de hidróxido de sódio $(\mathrm{NaOH})$ 0,5 mol L-1. A solução do balão de reação é filtrada para análises de metais $(\mathrm{Cd}, \mathrm{Cu}, \mathrm{Pb}, \mathrm{Ni}$ e $\mathrm{Zn})$. As soluções dos frascos receptores, que contêm os sulfetos (SVA), arrastados pelo gás nitrogênio, são transferidas para balões volumétricos de $100 \mathrm{~mL}$ e o volume aferido com água deionizada. Para a determinação do SVA utilizou-se o método azul de metileno. ${ }^{11}$ As medidas foram realizadas em um espectrômetro UV-VIS, Perkin Elmer Lambda 20 a um comprimento de onda de 678 $\eta \mathrm{m}$. Os resultados dos metais extraídos simultaneamente e dos sulfetos volatilizáveis por acidificação foram expressos em $\mu \mathrm{mol} \mathrm{g} \mathrm{g}^{-1}$ de peso seco.

A implantação do método para determinação da concentração de sulfetos volatilizáveis por acidificação (SVA) envolveu, numa primeira etapa, a realização de testes para avaliar a eficiência do sistema de captação. O teste foi realizado em duplicata utilizando solução padrão de sulfeto de sódio $\left(\mathrm{Na}_{2} \mathrm{~S}\right)$ a $5,91 \mu \mathrm{mol} \mathrm{L}{ }^{-1}$. As concentrações dos sulfetos foram avaliadas antes e depois do arraste com o nitrogênio. Recuperações com intervalos entre 80 e $120 \%$ são aceitáveis em testes desse tipo. Portanto, pode-se considerar que o método ofereceu uma recuperação satisfatória, uma vez que os resultados da recuperação ficaram entre 94 e $101 \%$.

\section{Levantamento e avaliação da estrutura da comunidade bentônica}

Os macroinvertebrados foram identificados taxonomicamente até a ordem e/ou família utilizando dados da Coleção de Macroinvertebrados Bentônicos do Laboratório de Ecologia de Bentos do ICB/UFMG. ${ }^{12}$
Avaliação e determinação da toxicidade aguda e crônica

Foram realizados testes de toxicidade crônica com Ceriodaphnia dubia e testes de toxicidade aguda com Daphnia similis em águas superficiais e águas de lixiviação dos sedimentos. Os testes foram realizados de acordo com os métodos propostos pela $\mathrm{ABNT}{ }^{13,14} \mathrm{O}$ ensaio consiste na exposição de jovens de Ceriodaphnia dubia e Daphnia similis a diferentes diluições da amostra, quais sejam 1, $5,15,25,50,75$ e $100 \%$, com o objetivo de avaliar os efeitos na sobrevivência e reprodução dos organismos.

Os resultados foram expressos em concentração efetiva de efeito não observado (CENO), que corresponde à maior concentração da amostra que não causa efeito deletério estatisticamente significativo na sobrevivência e reprodução dos organismos, nas condições de ensaio após 7 dias de experimento; e a concentração de efeito observado (CEO) é a menor concentração da amostra que causa efeito deletério estatisticamente significativo na sobrevivência e reprodução dos organismos, nas condições de ensaio.

\section{RESULTADOS E DISCUSSÃO}

\section{Análises físico-químicas}

Na Tabela 2 são mostrados os resultados dos parâmetros físicoquímicos obtidos nas estações de amostragem de águas superficiais, nos meses de fevereiro, agosto e novembro de 2006.

Em relação aos resultados dos parâmetros físico-químicos foi possível observar que os valores de oxigênio dissolvido e $\mathrm{pH}$ se apresentaram dentro do estabelecido na legislação ambiental. ${ }^{6}$ No entanto, os valores de sólidos totais dissolvidos ultrapassaram o limite legal $(500 \mathrm{mg} / \mathrm{L})$ na estação de amostragem localizada no córrego Lavagem, a montante de sua foz no ribeirão Espírito Santo (CL002). Ressalta-se ainda que os valores de condutividade elétrica também se apresentaram elevados no córrego Lavagem (acima de $500 \mu \mathrm{S}$ / $\mathrm{cm}$ ). Apesar de não existirem padrões legais de qualidade de água para a condutividade elétrica, em geral, níveis superiores a $100 \mu \mathrm{S}$ / cm indicam ambientes impactados. ${ }^{15}$ Esses resultados podem ser uma indicação do impacto ambiental associado à percolação de material da barragem de rejeitos.

\section{Análise da concentração de metais nas águas superficiais}

Na Tabela 3 são apresentados os resultados das médias e desviospadrão das concentrações dos metais cádmio, cobre, chumbo, níquel e zinco e os seus respectivos limites estabelecidos pela legislação ambiental para rios de Classe $2,{ }^{6}$ nas quatro estações de amostragem para as campanhas de novembro de 2005, agosto e novembro de 2006. Como pode ser observado, em todas as campanhas realizadas, os valores das concentrações dos metais avaliados nas amostras de águas superficiais ( $\mathrm{Cd}, \mathrm{Cu}, \mathrm{Pb}, \mathrm{Ni}$ e $\mathrm{Zn}$ ) estiveram abaixo do limite máximo estabelecido na legislação. ${ }^{6}$ Esses resultados podem estar relacionados com o fato bem conhecido de que concentrações de metais em água são frequentemente baixas, podendo flutuar drasticamente, dependendo do fluxo da água ou da intermitência das descargas de efluentes.

\section{Caracterização granulométrica}

Os resultados das análises granulométricas obtidos nos meses de fevereiro, agosto e novembro de 2006 são mostrados na Figura 1. Observa-se que os sedimentos são constituídos, principalmente, por areias média e fina, e a soma das frações silte e argila apresenta porcentagens maiores nas estações localizadas no ribeirão Espírito Santo (ES001, ES002 e ES003), principalmente na campanha realizada em fevereiro de 2006, apresentando respectivamente, 21,19; 21,75 e 
Tabela 2. Resultados dos parâmetros físico-químicos em água superficial

\begin{tabular}{|c|c|c|c|c|c|c|}
\hline $\begin{array}{l}\text { Pontos de } \\
\text { amostragem } \\
\end{array}$ & Data da coleta & $\begin{array}{l}\text { Temperatura } \\
\text { da água }\left({ }^{\circ} \mathrm{C}\right)\end{array}$ & $\begin{array}{l}\text { Condutividade } \\
\text { elétrica }(\mu \mathrm{S} / \mathrm{cm})\end{array}$ & $\begin{array}{c}\text { Oxigênio } \\
\text { dissolvido }(\mathrm{mg} / \mathrm{L})\end{array}$ & $\mathrm{pH}$ & $\begin{array}{c}\text { Sólidos totais } \\
\text { dissolvidos }(\mathrm{mg} / \mathrm{L})\end{array}$ \\
\hline \multirow[t]{3}{*}{ CLOO2 } & $\mathrm{fev} / 06$ & 26,7 & 556 & 5,6 & 6,7 & 616 \\
\hline & ago/06 & 17,3 & 1300 & 5,1 & 6,7 & 2160 \\
\hline & nov/06 & 25,2 & ---* & 6,4 & 6,7 & ---* \\
\hline \multirow[t]{3}{*}{ ES001 } & $\mathrm{fev} / 06$ & 27,7 & 26,7 & 5,2 & 5,8 & 29 \\
\hline & ago/06 & 23,2 & 29,4 & 7,4 & 6,7 & 49,9 \\
\hline & nov/06 & ---* & 17,6 & 7 & 6,4 & 9 \\
\hline \multirow[t]{3}{*}{ ES002 } & $\mathrm{fev} / 06$ & 29 & 29,7 & ---* & 6,6 & 33,5 \\
\hline & ago/06 & 21,8 & 29,6 & 6,5 & 6,7 & 50,1 \\
\hline & nov/06 & 26,8 & 19,1 & 8,5 & 6,35 & 9,7 \\
\hline \multirow[t]{3}{*}{ ES003 } & $\mathrm{fev} / 06$ & 28,4 & 29,9 & 5,9 & 6,4 & 32,9 \\
\hline & ago/06 & 23,1 & 27,9 & 6,7 & 6,7 & 47,8 \\
\hline & nov/06 & 26,4 & 20,8 & 6,6 & 6,4 & 10,6 \\
\hline \multicolumn{2}{|c|}{$\begin{array}{l}\text { Padrão CONAMA } 357 / 05 \text { para rios } \\
\text { de Classe } 2\end{array}$} & $---* *$ & $---* *$ & $>5$ & $6-9$ & 500 \\
\hline
\end{tabular}

* Não analisado. ** Não há limite na legislação.

Tabela 3. Resultados das análises de concentração de metais em água superficial

\begin{tabular}{|c|c|c|c|c|c|c|}
\hline \multirow{2}{*}{ Data da coleta } & \multirow{2}{*}{$\begin{array}{c}\text { Pontos de } \\
\text { amostragem }\end{array}$} & \multicolumn{5}{|c|}{ Concentração de metais em água superficial (mg/L) } \\
\hline & & $\mathrm{Cd}$ & $\mathrm{Pb}$ & $\mathrm{Cu}$ & $\mathrm{Zn}$ & $\mathrm{Ni}$ \\
\hline \multirow{4}{*}{ Nov/2005 } & CLO02 & $<0,001$ & $0,0016 \pm 0,0002$ & $*$ & $0,13 \pm 0,01$ & $<0,02$ \\
\hline & ES001 & $<0,001$ & $0,0067 \pm 0,0007$ & $*$ & $0,027 \pm 0,003$ & $<0,02$ \\
\hline & ES002 & $<0,001$ & $0,0066 \pm 0,0007$ & $*$ & $0,021 \pm 0,002$ & $<0,02$ \\
\hline & ES003 & $<0,001$ & $0,0086 \pm 0,0009$ & * & $0,027 \pm 0,003$ & $<0,02$ \\
\hline \multirow{4}{*}{ Ago/2006 } & CL002 & $<0,001$ & $<0,001$ & $<0,004$ & $0,032 \pm 0,003$ & $<0,02$ \\
\hline & ES001 & $<0,001$ & $<0,001$ & $<0,004$ & $0,033 \pm 0,003$ & $<0,02$ \\
\hline & ES002 & $<0,001$ & $<0,001$ & $<0,004$ & $0,032 \pm 0,003$ & $<0,02$ \\
\hline & ES003 & $<0,001$ & $<0,001$ & $<0,004$ & $0,028 \pm 0,003$ & $<0,02$ \\
\hline \multirow{4}{*}{ Nov/2006 } & CLO02 & $<0,001$ & $<0,001$ & $<0,004$ & $0,034 \pm 0,002$ & $<0,02$ \\
\hline & ES001 & $<0,001$ & $<0,001$ & $<0,004$ & $<0,02$ & $<0,02$ \\
\hline & ES002 & $<0,001$ & $<0,001$ & $<0,004$ & $<0,02$ & $<0,02$ \\
\hline & ES003 & $<0,001$ & $<0,001$ & $<0,004$ & $<0,02$ & $<0,02$ \\
\hline \multicolumn{2}{|c|}{$\begin{array}{l}\text { Padrão CONAMA 357/05 } \\
\text { para rios de Classe } 2\end{array}$} & 0,001 & 0,01 & 0,009 & 0,18 & 0,025 \\
\hline
\end{tabular}

* Não analisado.

$13,51 \%$. De maneira geral, os metais associam-se preferencialmente às frações finas dos sedimentos (silte e argila). ${ }^{16}$ No entanto, ao analisar os resultados não foi observada nenhuma correlação entre os dados de granulometria e metais nos sedimentos.

\section{Teor de matéria orgânica nos sedimentos}

Os sedimentos que contêm teores de matéria orgânica menores que $10 \%$ são classificados como inorgânicos. ${ }^{17}$ Sendo assim, os se- dimentos da região em estudo podem ser então classificados como inorgânicos, uma vez que todos os resultados obtiveram teores de matéria orgânica menores que 5\%.

A obtenção de baixos teores de matéria orgânica indica que, na possível presença de metais, esses sedimentos apresentam baixa capacidade de complexação com os mesmos, uma vez que a matéria orgânica pode se complexar com metais, tornando-os menos disponíveis para a biota. ${ }^{18}$ No entanto, não se verificou correlação entre os dados de matéria orgânica e metais nos sedimentos. 


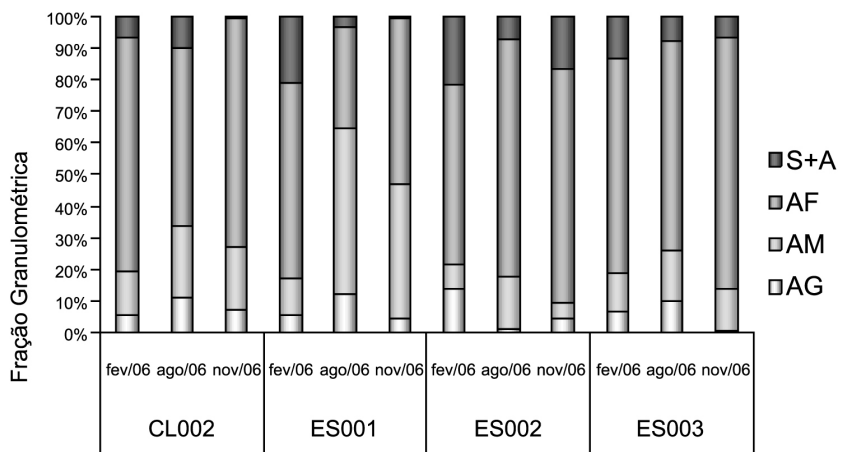

Figura 1. Resultados das análises granulométricas em amostras de sedimentos. $S+$ A: Silte mais Argila (diâmetro do grão $<0,06 \mathrm{~mm}$ ); AF: Areia fina (diâmetro do grão 0,06 < $<<0,2 \mathrm{~mm}$ ); AM: Areia média (diâmetro do grão $0,2<j<0,6 \mathrm{~mm}$ ); AG: Areia grossa (diâmetro do grão $0,6<j<2,0 \mathrm{~mm}$ )

\section{Aplicação dos valores-guia de qualidade de sedimentos}

\section{Valores-guia de qualidade de sedimentos do Canadá - VGQS canadenses}

Os valores-guia do Canadá são baseados em um banco de dados químicos e biológicos obtidos na América do Norte e elaborados sob patrocínio do Conselho Canadense de Ministérios do Meio Ambiente (CCME), o qual originou o Protocolo de Derivação dos Princípios Canadenses para a Qualidade dos Sedimentos e para a Proteção da Vida Aquática. ${ }^{2}$ A elaboração desse protocolo teve por objetivo estabelecer critérios para avaliação da qualidade dos sedimentos e do significado toxicológico das substâncias associadas aos mesmos para os organismos aquáticos.

Os VGQS canadenses foram baseados em bancos de dados de efei- tos e não efeitos, nesse caso, foram derivados dois valores-guia o TEL (threshold effect level) e o PEL (probable effect level). Esses valores-guia são valores numéricos de concentração de contaminantes individuais inorgânicos (metais e metalóides) e orgânicos (um amplo número de diferentes compostos) estatisticamente definidos com base em associações de valores da concentração química desses contaminantes nos sedimentos aos resultados de testes de toxicidade (aguda e crônica) realizados com um grande número de diferentes organismos teste. ${ }^{2}$

A aplicação destes valores-guia no presente trabalho teve caráter meramente orientativo na busca de evidências da presença de contaminantes em concentrações capazes de causar efeitos deletérios, sobretudo, com relação à toxicidade para a biota.

Na Tabela 4 são apresentados os valores-guia canadenses (TEL e PEL) e os resultados das análises de concentração de metais em sedimentos, para as quatro estações de amostragem nas campanhas de novembro de 2005 e agosto e novembro de 2006.

Como pode ser observado, os limites de TEL e PEL não foram violados em nenhum dos resultados obtidos, apresentando resultados de Cd no intervalo <0,05-0,17 mg/kg, Cu $<1-14$ mg/kg, $\mathrm{Pb}<10-16$ $\mathrm{mg} / \mathrm{kg}, \mathrm{Ni}<5-17 \mathrm{mg} / \mathrm{kg}$ e Zn $<3-59 \mathrm{mg} / \mathrm{kg}$. Contudo, apenas o ponto localizado no córrego Lavagem, a montante de sua foz no ribeirão Espírito Santo (CL002), apresentou valores de concentração dos metais avaliados acima dos valores obtidos no ponto de "referência" (ES001). O ponto CL002 corresponde justamente àquele onde é esperada uma maior interferência do material percolado da barragem de rejeitos.

Valores-guia baseados na teoria do equilíbrio de partição

O SVA é uma fase controladora de cátions metálicos divalentes nos sedimentos. Em sedimentos não contaminados, o SVA é representado principalmente por monossulfetos de ferro e na presença de alguns cátions metálicos $(\mathrm{Cd}, \mathrm{Cu}, \mathrm{Pb}, \mathrm{Ni}$ e $\mathrm{Zn})$ os monossulfetos de ferro reagem com esses cátions formando sulfetos metálicos

Tabela 4. Resultados da aplicação dos valores-guia de qualidade de sedimentos canadenses

\begin{tabular}{|c|c|c|c|c|c|c|}
\hline \multirow[t]{2}{*}{ Data da coleta } & \multirow{2}{*}{$\begin{array}{c}\text { Pontos de } \\
\text { amostragem }\end{array}$} & \multicolumn{5}{|c|}{ Concentração de metais nas amostras de sedimentos (mg/kg) } \\
\hline & & $\mathrm{Cd}$ & $\mathrm{Pb}$ & $\mathrm{Cu}$ & $\mathrm{Zn}$ & $\mathrm{Ni}$ \\
\hline \multirow{4}{*}{ Nov/2005 } & CL002 & $0,17 \pm 0,02$ & $<10$ & $8,0 \pm 1,5$ & $58,5 \pm 2$ & $<5$ \\
\hline & ES001 & $<0,05$ & $<10$ & $3,2 \pm 0,5$ & $3,3 \pm 0,3$ & $<5$ \\
\hline & ES002 & $<0,05$ & $<10$ & $2,3 \pm 0,5$ & $3,2 \pm 0,3$ & $<5$ \\
\hline & ES003 & $<0,05$ & $<10$ & $2,0 \pm 0,4$ & $3,4 \pm 0,5$ & $<5$ \\
\hline \multirow{4}{*}{ Ago/2006 } & CLO02 & $0,085 \pm 0,015$ & $15,5 \pm 1,5$ & $12 \pm 0,5$ & $58,5 \pm 4,5$ & $17 \pm 2$ \\
\hline & ES001 & $<0,05$ & $<10$ & $6 \pm 0,5$ & $<10$ & $6,5 \pm 0,5$ \\
\hline & $\mathrm{ES} 002$ & $<0,05$ & $<10$ & $3 \pm 0,4$ & $<10$ & $7,0 \pm 0,5$ \\
\hline & ES003 & $<0,05$ & $<10$ & $<1$ & $<10$ & $<5$ \\
\hline \multirow{8}{*}{ Nov/2006 } & CL002 & $0,10 \pm 0,02$ & $16 \pm 1,5$ & $14,0 \pm 0,5$ & $58 \pm 2$ & $13,0 \pm 1,5$ \\
\hline & ES001 & $<0,05$ & $<10$ & $3,5 \pm 0,3$ & $12,0 \pm 0,2$ & $6,0 \pm 0,5$ \\
\hline & ESO02 & $<0,05$ & $<10$ & $5,5 \pm 0,2$ & $14,0 \pm 0,3$ & $7,5 \pm 0,2$ \\
\hline & ES003 & $<0,05$ & $<10$ & $2,4 \pm 0,2$ & $10,0 \pm 0,1$ & $<5$ \\
\hline & & \multicolumn{5}{|c|}{ Valores-guia de qualidade de sedimentos canadenses (mg/kg) } \\
\hline & & $\mathrm{Cd}$ & $\mathrm{Pb}$ & $\mathrm{Cu}$ & $\mathrm{Zn}$ & $\mathrm{Ni}$ \\
\hline & TEL & 0,6 & 35 & 35,7 & 123 & 18 \\
\hline & PEL & 3,5 & 91,3 & 197 & 316 & 36 \\
\hline
\end{tabular}


insolúveis. Portanto, na presença de SVA, metais como $\mathrm{Cd}, \mathrm{Cu}, \mathrm{Pb}$, Ni e Zn são retirados da coluna d'água e das águas intersticiais por precipitação, com o SVA presente nos sedimentos. ${ }^{3}$ Diferentemente dos metais totais, a fase metálica que é solubilizada pelo método descrito por: Allen et al. ${ }^{10}$ é a fração de metais associada aos SVA, aos carbonatos, aos óxidos amorfos, adsorvidos às superfícies das partículas sedimentares e frações mais lábeis da matéria orgânica nos sedimentos totais, denominada metais extraídos simultaneamente (MES).

De acordo com essa teoria, a Agência de Proteção Ambiental dos Estados Unidos desenvolveu um método para avaliação da qualidade dos sedimentos por meio da comparação direta da soma da concentração molar dos cinco metais ( $\mathrm{Cd}, \mathrm{Cu}, \mathrm{Pb}, \mathrm{Ni}$ e $\mathrm{Zn})$ e a concentração do SVA, presentes nos sedimentos, extraídos simultaneamente pelo método descrito por: Allen et al.. ${ }^{10} \mathrm{~A}$ toxicidade para organismos aquáticos não tem sido observada em ensaios de ecotoxicidade em sedimentos que apresentam $\Sigma$ MES - SVA $\leq 0$. Entretanto, se $\Sigma$ MES - SVA > 0, o sedimento não necessariamente irá apresentar toxicidade. Nesse caso, pode haver o controle de outra fase, por exemplo, a do carbono orgânico total (COT). Portanto, nesse caso deverá ser feita a correção pelo COT. O modelo ( $2 \mathrm{MES}$ - SVA)/COT é conceitualmente simples, entretanto, tem sido utilizado com grande sucesso para predizer sobre a não toxicidade dos sedimentos. Para predizer se o sedimento é realmente tóxico, outros critérios também devem ser aplicados. Nesse caso, recomenda-se o uso conjunto de ( $\mathrm{M}$ MES - SVA)/COT e os valores-guia de unidades tóxicas das águas intersticiais (VGUTAI). Em sedimentos oxidantes, a biodisponibilidade dos metais é controlada por outras fases ligantes, tais como carbono orgânico particulado, íons carbonato e óxidos de ferro e manganês. ${ }^{3}$

Os resultados das análises de metais em sedimentos para cada estação de amostragem e a aplicação dos valores-guia de qualidade de sedimentos baseados na teoria do equilíbrio de partição - TEP, obtidos para as campanhas de agosto e novembro de 2006 são apresentados na Tabela 5.

Pode-se observar que a presença de zinco nas duas campanhas de amostragem na estação localizada no córrego Lavagem, a montante de sua foz no ribeirão Espírito Santo (CL002) foi a mais representativa, em relação aos demais metais e demais pontos de amostragem. No entanto, nesse ponto, foi obtido $\Sigma \mathrm{MES}$ - SVA $<0$, o que indica que os sedimentos estudados não esgotaram seu potencial de retenção de metais na forma de sulfetos, neste ponto de amostragem. Os valores dos metais nas outras estações de amostragem - ES001, ES002 e ES003 - apresentaram-se muito próximos aos limites de detecção, apresentando baixa confiabilidade dos resultados para serem considerados. As faixas de concentração do somatório dos metais extraídos simultaneamente ficaram entre 0,02 e $1,30 \mu \mathrm{mol} / \mathrm{g}$, e os sulfetos volatilizáveis por acidificação apresentaram concentrações entre 0,03 $19,40 \mu \mathrm{mol} / \mathrm{g}$. Ressalta-se que os resultados obtidos de SVA e MES no presente trabalho apresentaram-se muito inferiores aos obtidos na represa Billings em São Paulo - SP, quando foi encontrado $\sum \mathrm{MES}$ igual a $17,9 \mu \mathrm{mol} / \mathrm{g}$ e SVA de $64,9 \mu \mathrm{mol} / \mathrm{g} .{ }^{19}$ Contudo, os ambientes são totalmente distintos, tanto em relação à morfologia quanto aos diferentes fatores de pressão ambiental.

Assim sendo, segundo a avaliação dos VGQS baseados na teoria do equilíbrio de partição, recomendada pela USEPA, os metais presentes nos sedimentos não estão disponibilizados para a biota nas estações de amostragem avaliadas. Contudo, nas estações localizadas no ribeirão Espírito Santo - ES001, ES002 e ES003 - a proporção entre MES e SVA se aproxima de 1,0. Portanto, caso ocorra um aumento no aporte destes metais nesses trechos, o sistema não parece ser capaz de imobilizar níveis de SVA capazes de manter este maior aporte na fase sólida. Sendo assim, seria interessante a continuidade desse trabalho com o intuito de monitorar a biodisponibilidade dos metais, principalmente nesses trechos, ou, até mesmo, avaliar a existência de outras fases sedimentares envolvidas no controle da biodisponibilidade dos metais, por exemplo, óxidos ou matéria orgânica.

\section{Avaliação da estrutura da comunidade bentônica}

O índice de diversidade de Shannon-Wiener, a equitabilidade de Pielou, a densidade (número de indivíduo por $\mathrm{m}^{2}$ ) e a riqueza taxonômica foram calculados de forma a se obter subsídios para o tratamento dos dados relativos à estrutura da comunidade bentônica na região de estudo.

Os resultados apresentados na Tabela 6 mostram o ponto adotado como referência (ES001), localizado no ribeirão Espírito Santo, a montante da confluência com o córrego Lavagem, como o trecho que apresenta maior riqueza, equitabilidade e diversidade de espécies encontradas, o que indica que esse trecho se encontra bem preservado em relação aos trechos em estudo.

$\mathrm{O}$ valor de equitabilidade do ponto (ES001) foi igual a 0,62, indicando que nesse trecho existe uma distribuição mais homogênea entre as famílias encontradas em relação aos outros trechos avaliados. Da mesma forma, o valor de diversidade nesse trecho apresentou resultados mais elevados indicando, portanto, que existe uma proporcionalidade entre as famílias das espécies encontradas.

A análise de similaridade entre os trechos foi apresentada em forma de dendrograma; essa análise utiliza uma associação dos dados de diversidade, equitabilidade e dominância das diferentes estações de amostragem. Os resultados das análises são mostrados na Figura 2.

Como pode ser observado, existe uma alta similaridade entre os pontos CL002 e ES002. A análise dos resultados obtidos indica visivelmente a influência direta da barragem de rejeitos sobre a estrutura da

Tabela 5. Resultados dos valores-guia baseados na teoria do equilíbrio de partição - TEP (valores expressos em $\mu \mathrm{mol} / \mathrm{g}$ )

\begin{tabular}{|c|c|c|c|c|c|c|c|c|c|}
\hline $\begin{array}{l}\text { Data da } \\
\text { coleta }\end{array}$ & $\begin{array}{c}\text { Pontos de } \\
\text { amostragem }\end{array}$ & $\mathrm{Cd}$ & $\mathrm{Cu}$ & $\mathrm{Pb}$ & $\mathrm{Ni}$ & $\mathrm{Zn}$ & $\Sigma[\mathrm{MES}]$ & SVA & $\begin{array}{c}\Sigma[\mathrm{MES}]- \\
\text { SVA }\end{array}$ \\
\hline \multirow{6}{*}{$\begin{array}{l}\stackrel{8}{0} \\
\stackrel{80}{<}\end{array}$} & CLO02 & $<0,001$ & $\mathbf{0 , 0 8 0} \pm 0,006$ & 0,1 & $\mathbf{0 , 0 9 0} \pm 0,001$ & $\mathbf{1 , 0 7} \pm 0,1$ & 1,3 & $8 \pm 3$ & $-7,07$ \\
\hline & ES001 & $<0,001$ & $0,030 \pm 0,004$ & $<0,01$ & $0,020 \pm 0,001$ & $<0,01$ & 0,05 & $0,04 \pm 0,01$ & 0,01 \\
\hline & ES002 & $<0,001$ & $0,040 \pm 0,006$ & $<0,01$ & $0,030 \pm 0,001$ & $0,03 \pm 0,02$ & 0,11 & $0,52 \pm 0,16$ & $-0,4$ \\
\hline & ES003 & $<0,001$ & $<0,01$ & $<0,01$ & $0,020 \pm 0,002$ & $<0,01$ & 0,03 & $0,03 \pm 0,01$ & 0 \\
\hline & CLOO2 & $<0,001$ & $\mathbf{0 , 0 6} \pm 0,01$ & $\mathbf{0 , 0 6 0} \pm 0,001$ & $\mathbf{0 , 1 2} \pm 0,02$ & $\mathbf{0 , 9 2} \pm 0,02$ & 1,15 & $19 \pm 1$ & $-18,25$ \\
\hline & ES001 & $<0,001$ & $0,020 \pm 0,002$ & $<0,01$ & $0,020 \pm 0,001$ & $<0,01$ & 0,04 & $0,06 \pm 0,04$ & $-0,02$ \\
\hline \multirow{2}{*}{$\frac{\wp}{o}$} & ES002 & $<0,001$ & $0,030 \pm 0,001$ & $<0,01$ & $0,030 \pm 0,005$ & $<0,01$ & 0,06 & $0,08 \pm 0,04$ & $-0,02$ \\
\hline & ES003 & $<0,001$ & $0,020 \pm 0,002$ & $<0,01$ & $<0,01$ & $<0,01$ & 0,02 & $0,03 \pm 0,02$ & $-0,01$ \\
\hline
\end{tabular}


Tabela 6. Resultados da diversidade da fauna de insetos aquáticos

\begin{tabular}{lcccc}
\hline & \multicolumn{4}{c}{ Pontos de amostragem } \\
& CL002 & ES001 & ES002 & ES003 \\
\hline Riqueza total & 23 & 27 & 21 & 19 \\
Densidade total & 4089 & 6096 & 8433 & 1844 \\
Equitabilidade & 0,36 & 0,62 & 0,19 & 0,36 \\
Diversidade & 1,11 & 2,04 & 0,58 & 1,07 \\
\hline
\end{tabular}

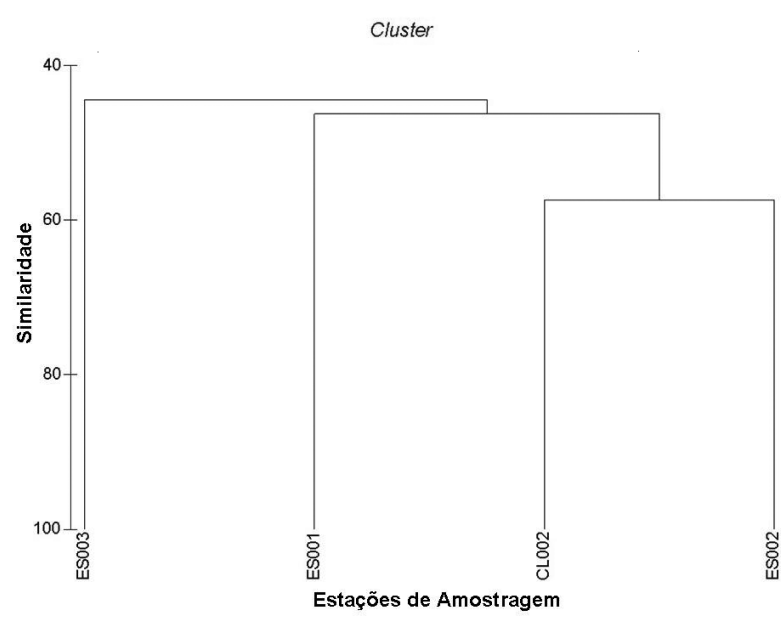

Figura 2. Dendograma de similaridade de Bray-Curtis, elaborado a partir dos dados de diversidade, equitabilidade e dominância das amostras de macroinvertebrados bentônicos

comunidade bentônica nos trechos localizados no córrego Lavagem, a montante de sua foz no ribeirão Espírito Santo (CL002), e no ribeirão Espírito Santo, a jusante da confluência com o córrego Lavagem (ES002).

\section{Resultados dos ensaios ecotoxicológicos nas amostras de águas superficiais e sedimentos}

\section{Toxicidade crônica}

Os resultados dos testes de toxicidade crônica para o microcrustáceo Ceriodaphnia dubia em águas superficiais e nas águas de lixiviação dos sedimentos são apresentados nas Tabelas 7 e 8 , respectivamente.

Como pode ser verificado houve ocorrência de efeitos tóxicos crônicos para o microcrustáceo Ceriodaphnia dubia nas amostras de águas superficiais nas três estações de monitoramento localizadas no ribeirão Espírito Santo - ES001, ES002 e ES003. Com relação à frequência de ocorrência de efeitos tóxicos crônicos para o microcrustáceo Ceriodaphnia dubia nas águas de lixiviação dos sedimentos, pode-se inferir que os organismos teste indicaram a existência de toxicidade nas águas de lixiviação dos sedimentos nos trechos localizados no ribeirão Espírito Santo, a montante de sua foz no rio São Francisco (ES003) e no córrego Lavagem, a montante de sua foz no ribeirão Espírito Santo (CL002).

Em suma, os resultados dos testes de ecotoxicidade demonstraram a ocorrência de efeitos tóxicos crônicos em todos os trechos de monitoramento, seja nas águas superficiais, seja nas águas de lixiviação dos sedimentos. Sendo assim, pode haver a presença de outras substâncias não identificadas, inclusive no ponto de referência, que podem interferir nos processos de reprodução e crescimento dos organismos teste. Esses resultados podem estar relacionados ao desenvolvimento de atividades de agricultura existentes na região e, portanto, ao uso de agrotóxicos e fertilizantes.
Tabela 7. Ocorrência de toxicidade crônica para o microcrustáceo Ceriodaphnia dubia nas amostras de águas superficiais

\begin{tabular}{|c|c|c|}
\hline $\begin{array}{l}\text { Pontos de } \\
\text { amostragem }\end{array}$ & ago/06 & nov/06 \\
\hline CL002 & NA* & NA \\
\hline ES001 & $\begin{array}{l}\mathrm{CENO} * *=0,5 \% \\
\text { da amostra } \\
\text { CEO } * * *=1 \% \\
\text { da amostra }\end{array}$ & $\begin{array}{l}\mathrm{CENO}=5 \% \text { da amostra } \\
\mathrm{CEO}=15 \% \text { da amostra }\end{array}$ \\
\hline ESO02 & $\begin{array}{l}\mathrm{CENO}=25 \% \\
\text { da amostra } \\
\mathrm{CEO}=50 \% \\
\text { da amostra }\end{array}$ & $\begin{array}{l}\text { CENO }=5 \% \text { da amostra } \\
\text { CEO }=15 \% \text { da amostra }\end{array}$ \\
\hline ES003 & NA & $\begin{array}{l}\text { CENO }=75 \% \text { da amostra } \\
\mathrm{CEO}=100 \% \text { da amostra }\end{array}$ \\
\hline \multicolumn{3}{|c|}{$\begin{array}{l}* \mathrm{NA}=\text { Não apresentou toxicidade. } * * \mathrm{CENO}=\text { Concentração } \\
\text { efetiva de efeito não observado. } * * * \mathrm{CEO}=\mathrm{Concentração} \mathrm{de} \mathrm{efeito} \\
\text { observado. }\end{array}$} \\
\hline \multicolumn{3}{|c|}{$\begin{array}{l}\text { Tabela 8. Ocorrência de toxicidade crônica com o microcrustáceo } \mathrm{Ce} \\
\text { riodaphnia dubia nas águas de lixiviação das amostras de sedimento }\end{array}$} \\
\hline $\begin{array}{l}\text { Pontos de } \\
\text { amostragem }\end{array}$ & ago/06 & nov/06 \\
\hline CL002 & NA* & $\begin{array}{l}\text { CENO }=25 \% \text { da amostra } \\
\text { CEO }=50 \% \text { da amostra }\end{array}$ \\
\hline ES001 & NA & NA \\
\hline ES002 & NA & NA \\
\hline ES003 & $\begin{array}{l}\text { CENO } * *=1 \% \\
\text { da amostra } \\
\text { CEO } * * *=5 \% \\
\text { da amostra }\end{array}$ & $\begin{array}{l}\mathrm{CENO}=75 \% \text { da amostra } \\
\mathrm{CEO}=100 \% \text { da amostra }\end{array}$ \\
\hline
\end{tabular}

* NA = Não apresentou toxicidade. $* *$ CENO = Concentração efetiva de efeito não observado e $* * * \mathrm{CEO}=$ Concentração de efeito observado.

\section{Toxicidade aguda}

Com relação à frequência de ocorrência de efeitos tóxicos agudos para o microcrustáceo Daphnia similis não foi observado efeito adverso em nenhuma das amostras de águas superficiais e de lixiviação dos sedimentos.

\section{CONCLUSÕES}

Em relação às águas superficiais, apenas o resultado do parâmetro sólido total dissolvido na estação localizada no córrego Lavagem (CL002) apresentou um valor acima do estabelecido pela Resolução CONAMA 357/05. ${ }^{6}$ Os demais parâmetros apresentaram-se dentro dos limites legais.

Os resultados das análises granulométricas mostraram que, em todas as estações de amostragem, os sedimentos constituem predominantemente de areia fina. Além disso, foi verificado que o teor de matéria orgânica em todas as estações é baixo $(<5 \%)$, indicando que os sedimentos avaliados podem ser classificados como inorgânicos.

Os sedimentos da estação CL002 apresentaram valores de metais totais acima dos valores obtidos no ponto de referência, indicando que essa estação apresenta indícios de impactos decorrentes da barragem de rejeitos presente nas cabeceiras desse córrego.

A avaliação da recuperação do SVA mostrou que o método ofere- 
ceu um resultado satisfatório, uma vez que os valores de recuperação obtidos ficaram entre 94 e 101\%. Foi possível observar a presença, principalmente, de zinco nas duas campanhas de amostragem na estação localizada no córrego Lavagem, a montante de sua foz no ribeirão Espírito Santo (CL002) nas amostras de sedimentos. Entretanto, obteve-se $\sum$ MES - SVA $<0$, indicando que os sulfetos se encontram em níveis suficientes para prevenir a biodisponibilidade dos metais.

A análise dos macroinvertebrados bentônicos indicou que o ponto de referência localizado no ribeirão Espírito Santo, a montante da confluência com o córrego Lavagem (ES001), é a estação mais preservada entre àqueles do presente estudo. A análise de similaridade entre os trechos demonstrou alta similaridade entre as estações localizadas no córrego Lavagem, a montante de sua foz no ribeirão Espírito Santo (CL002) e no ribeirão Espírito Santo, a jusante da confluência com o córrego Lavagem (ES002), sendo essas as estações mais alteradas devido à influência direta da barragem de rejeitos sobre a estrutura da comunidade bentônica nesses trechos.

Os ensaios ecotoxicológicos mostraram que todos os trechos apresentam condições adversas que poderão interferir nos processos de reprodução e crescimento para o microcrustáceo Ceriodaphnia dubia em todos os trechos de monitoramento, seja nas águas superficiais, seja nas águas de lixiviação dos sedimentos. Portanto, as condições de toxicidade podem ser provenientes também do desenvolvimento de atividades de agricultura na região. Essas, por sua vez, estão associadas ao uso de fertilizantes e agrotóxicos em quantidades tais que podem comprometer a qualidade das águas e dos sedimentos. ${ }^{20}$

Os resultados permitiram verificar que trechos da sub-bacia em estudo, os quais recebem influência direta da barragem de rejeitos, ainda não se encontram contaminados em níveis tais que possam estar causando efeitos deletérios agudos à biota, conforme esperado de acordo com os resultados da relação entre SVA e MES, pois ocorreram níveis de SVA superiores aos dos metais simultaneamente extraídos. Entretanto, pode-se afirmar que esses trechos encontram-se alterados em relação ao ponto adotado como referência.

No sentido de complementar este estudo, sugere-se que sejam realizados experimentos adicionais com o objetivo de se obter mais dados e chegar a respostas mais conclusivas a respeito dos possíveis impactos ambientais associados à percolação de efluentes da barragem de rejeitos, o que implica na continuidade ao programa de monitoramento implantado com o objetivo de acompanhar a ocorrência de alterações no sistema aquático em estudo.

\section{AGRADECIMENTOS}

Ao Instituto Mineiro de Gestão das Águas, IGAM, pelo apoio financeiro, sem o qual este trabalho não poderia ter sido efetivado. Ao Prof. Dr. A. A. Mozeto e sua aluna de mestrado Erida, pelo apoio técnico na realização das análises de SVA/MES. Ao Prof. M. C. de F. Pereira e ao técnico W. R. Ferreira, pelo apoio nas coletas e análise dos macroinvertebrados bentônicos.

\section{REFERÊNCIAS}

1. Manahan, S. E.; Environmental Chemistry, $5^{\text {th }}$ ed., Lewis: Michigan, 1991.

2. Nascimento, M. R. L.; Mozeto, A. A.; Soil and Sediment Contamination 2008, 17, 269.

3. Environmental Protection Agency; Procedures for the Derivation of Equilibrium Partitioning Sediment Benchmarks (ESBs) for the Protection of Benthic Organisms: Metal mixtures (Cadmium, Copper, Lead, Nickel, Silver, and Zinc), USEPA: Washington, 2005.

4. De Almeida, C. A.; Rocha, O.; J. Braz. Soc. Ecotoxicol. 2006, 1, 141.

5. Chapman, P. M.; Wang, F.; Environ. Sci. Technol. 1999, 33, 3937.

6. Resolução CONAMA No 357 de março de 2005; dispõe sobre a classificação dos corpos de água e diretrizes ambientais para o seu enquadramento, bem como estabelece as condições e padrões de lançamento de efluentes, e dá outras providências, Diário Oficial da União, Seção I, 17/03/2005, Brasília.

7. http://www.epa.gov/osw/hazard/testmethods/sw846/pdfs/3050b.pdf, acessada em Maio 2007.

8. Macedo, J. A. B.; Métodos Laboratoriais de Análises: Físico-Químicas e Microbiológicas, $2^{\mathrm{a}}$ ed., CRQ: Belo Horizonte, 2003.

9. Associação Brasileira de Normas Técnicas; NBR 6502; Rochas e solos, Rio de Janeiro, 1995

10. Allen, H. E.; FU, G.; Deng, B.; Environm. Toxicol. Chem. 1992, 12, 1441.

11. Rao, C. N. R.; Ultra-Violet and Visible Spectroscopy: chemical applications, $2^{\text {nd }}$ ed., Butterworths: London, 1967.

12. Merritt, R. W.; Cummins, K. W.; An Introduction to the Aquatic Insects of North America, $2^{\text {nd }}$ ed., Kendall/Hunt Publishing: Dubuque, 1988; Callisto, M.; Barbosa, F. A. R.; Vianna, J. A.; Anais do $4^{\circ}$ Simpósio de Ecossistemas Brasileiros, Águas de Lindóia, Brasil, 1998.

13. Associação Brasileira de Normas Técnicas; NBR 13373, 2005

14. Associação Brasileira de Normas Técnicas; NBR 2713, 2004.

15. http://www.cetesb.sp.gov.br/Agua/rios/variaveis.asp\#condutividade, acessada em Fevereiro 2008.

16. Föstner, U; Salomons, W.; Environ. Technol. Lett. 1988, 1, 495.

17. Esteves, F. A.; Fund. Limn., $2^{\mathrm{a}}$ ed., Interciência: Rio de Janeiro, 1998.

18. Brigante, J.; Espíndola, E. L. G.; Limnologia fluvial, RiMa: São Carlos, 2003.

19. Silvério, P. F.; Tese de Doutorado, Universidade Federal de São Carlos, Brasil, 2003.

20. Machado, A. T. M.; Estudos Avançados 2008, 22. 